\title{
Synthesis, Photochromic Property and Application for Optical Recording of a New Asymmetrical Diarylethene
}

\author{
Huirong Du ${ }^{1,2}$, Shangfen Gao ${ }^{1 *}$, Li Yang ${ }^{2}$ \\ ${ }^{1}$ Department of Chemistry and Chemical Engineering, Sichuan University of Arts and Science, Dazhou, China \\ ${ }^{2}$ Sichuan Higher Education Key Laboratory of Characteristic Plant Development and Research, \\ Sichuan University of Arts and Science, Dazhou, China \\ Email: ${ }^{*}$ shangfengao@163.com
}

Received October 26, 2012; revised November 27, 2012; accepted December 14, 2012

\begin{abstract}
The reversible molecule switching of an assymmetrical photochromic diarylethene 1-[2-methy1-5-(3-chlorophenyl)-3thienyl]-2-(1,2-dimethy1-3-indol) perfluorocyelopentene (1a) has been prepared for the examination of photo-switching, fluorescence switching. This compound exhibited reversible photochromism, changing from colorless to blue after irradiation with UV light both in solution and in poly-methyl methacrylate (PMMA) amorphous film. Also, it exhibited remarkable fluorescence switching in the solid state. Using diarylethene 1b/PMMA film as recording medium, polarization optical recording was performed perfectly by a He-Ne laser with $633 \mathrm{~nm}$ wavelength. The results demonstrated that it can be potentially used as polarization optical recording medium.
\end{abstract}

Keywords: Diarylethene; Fluorescence; Optical Recording

\section{Introduction}

Photochromic materials have been extensively investigated for their potential applications in erasable optical memories, displays, and optical switches [1]. The properties of photochromic materials, such as their absorption spectrum, refractive index, dielectric constant, oxidation and reduction potentials, photoluminescence, dipole moment, and ionization potential (Ip), can be changed reversibly during the photochromic process. This unique character can be applied to different types of photonic devices [2-5]. Among the photochromic compounds, diarylethenes are regarded as the best candidates for such devices [1], because of their advantages such as a high efficiency of photoisomerizations, sufficient thermal stability of both the open and the close forms, a very high resistance to photofatigue, and the ease with which the reaction can be monitored by UV-vis spectroscopy [6-9]. These differences have been utilized to control their functions such as electrical conductivity, alignment of liquid crystals, light-driven organogelators, and photocontrolled release and uptake.

Among diarylethene derivatives so far synthesized [10-13], most of the heteroaryl moieties have been thiophene or benzothiophene rings, and other heteroaryl

*Corresponding author. moieties, such as furan, thiazole, benzofuran, pyrrole and indene [1], have also been reported partially. But diarylethene bearing thiophene and indole moieties haven't been reported until now. In this paper, we have designed and prepared a new asymmetrical diarylethene derivative with thiophene and indole moieties. i.e., 1-[2-methy1-5(3-chlorophenyl)-3-thienyl]-2-(1,2-dimethy1-3-indol) perfluorocyelopentene (1a).

We synthesized asymmetrical diarylethenes having an indole moiety, and the compound showed good thermal stability and strong fatigue resistance in the solid state. The results that asymmetrical diarylethene with an indole moieties have better thermal stability and stronger fatigue resistance than the symmetrical one are very interesting and important. Its photochromic reactivity, fluorescence property and application for optical recording were investigated. The photochromic reaction of diarylethene 1a is shown in Scheme 1.

\section{Experimental}

The synthetic route for diarylethene 1a is shown in Scheme 2. It was synthesized according to the similar procedure of $\mathrm{Pu}$ et al. [12]. The structure of compound 1a was confirmed by ${ }^{1} \mathrm{H}$ NMR spectroscopy, IR and ${ }^{13} \mathrm{C}$ NMR spectroscopy. ${ }^{1} \mathrm{H}$ NMR (400 $\left.\mathrm{MHz}, \mathrm{CDCl}_{3}\right): \delta 1.80$ $\left(\mathrm{s}, 3 \mathrm{H},-\mathrm{CH}_{3}\right), 2.04\left(\mathrm{~s}, 3 \mathrm{H},-\mathrm{CH}_{3}\right), 3.65\left(\mathrm{~s}, 3 \mathrm{H},-\mathrm{CH}_{3}\right)$, 


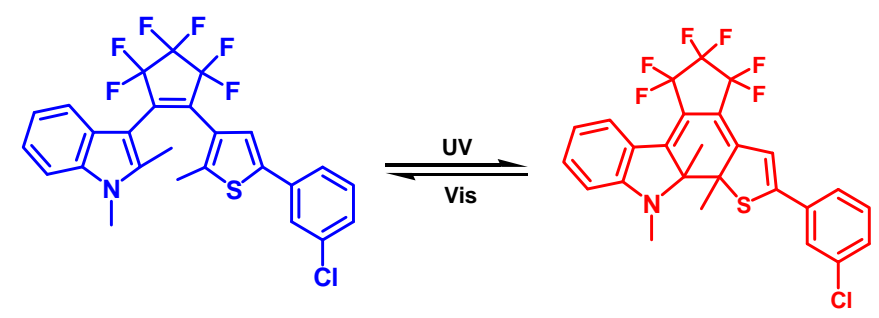

Scheme 1. Photochromism of diarylethenes 1a.

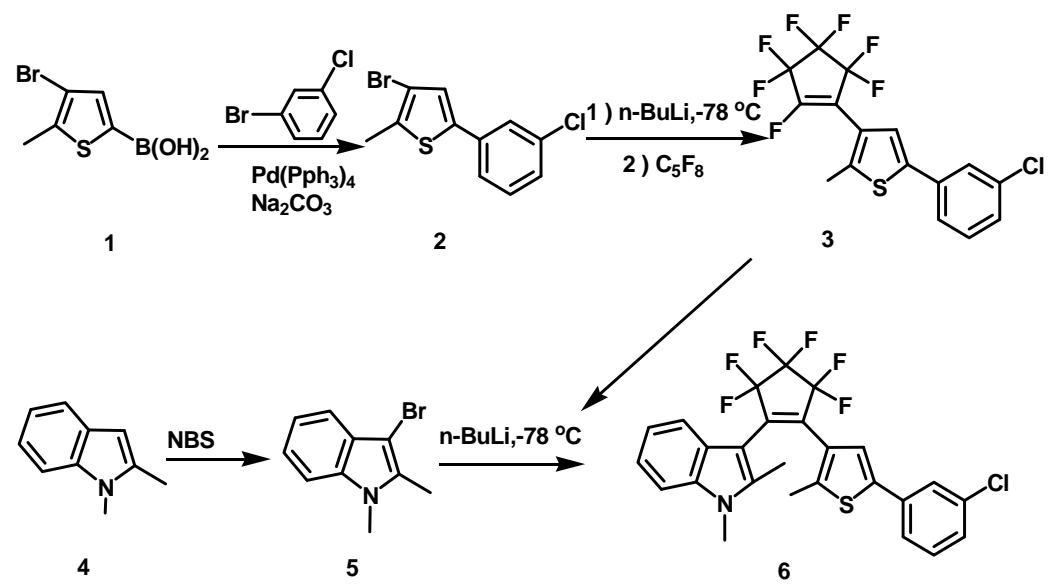

Scheme 2. Synthetic route for the compound 1a.

$7.14(\mathrm{t}, 1 \mathrm{H}, J=8.0 \mathrm{~Hz}$, thiophene-H), $7.22(\mathrm{~d}, 1 \mathrm{H}, J=$ $8.0 \mathrm{~Hz}$, phenyl-H), 7.29 (d, $1 \mathrm{H}, J=8.0 \mathrm{~Hz}$, phenyl-H), 7.39 (s, 1H, phenyl-H), $7.48(\mathrm{t}, 1 \mathrm{H}, J=8.0 \mathrm{~Hz}$, phenyl$\mathrm{H}), 7.56(\mathrm{t}, 2 \mathrm{H}, J=7.6 \mathrm{~Hz}$, phenyl-H), $7.74(\mathrm{t}, 2 \mathrm{H}, J=$ $8.0 \mathrm{~Hz}$, phenyl-H); ${ }^{13} \mathrm{C}$ NMR $\left(400 \mathrm{MHz}, \mathrm{CDCl}_{3}\right): \delta 11.46$, $14.21,30.01,109.21,113.37,116.50,118.38,119.50$, $122.14,124.42,125.57,127.17,128.90,129.59,129.84$, $130.81,134.89,137.13,138.82,142.35$; IR $\left(\mathrm{KBr}, \mathrm{cm}^{-1}\right)$ : 752, 779, 812, 840, 869, 893, 972, 997, 1020, 1047, 1078, $1112,1161,1182,1276,1340,1400,1458,1624,1668$, 3170. Calcd for $\mathrm{C}_{26} \mathrm{H}_{19} \mathrm{ClF}_{6} \mathrm{NS}(\%)$ : Calcd C, 59.38; H, 3.45; Cl, 6.74; N, 2.66; S, 6.10. Found C, 59.13; H, 3.34; $\mathrm{Cl}, 6.80 ; \mathrm{N}, 2.74 ; \mathrm{S}, 6.24 ; \mathrm{mp}: 143.5^{\circ} \mathrm{C}-145.3^{\circ} \mathrm{C}$.

The recording film was prepared as follows: diarylethene $1 \mathbf{a}(10 \mathrm{mg})$ was dissolved ultrasonically in PMMAchloroform solution $(10 \%, \mathrm{w} / \mathrm{w}, 1 \mathrm{ml})$. The mixture solution was then spin coated on a glass substrate $(20 \mathrm{~mm} \times$ $20 \mathrm{~mm} \times 1 \mathrm{~mm}$ ) which was then dried in air at room temperature. The thickness of the film was about $12 \mu \mathrm{m}$. The sample was colored homogeneously by irradiation with $313 \mathrm{~nm}$ UV light to convert the open-ring isomer (1a) to the closed-ring isomer (1b) before being recorded.

\section{Results and Discussion}

\subsection{Photochromism of Diarylethene}

The diarylethene 1a exhibits good photochromism both in hexane solution $\left(2 \times 10^{-5} \mathrm{~mol} \cdot \mathrm{L}^{-1}\right)$ and in PMMA amorphous film $(10 \% \mathrm{w} / \mathrm{w})$. As shown in Figure 1, in hexane solution, the absorption maximum of compound 1 a was observed at $220 \mathrm{~nm}\left(\varepsilon=5.5 \times 10^{4} \mathrm{~L} \cdot \mathrm{mol}^{-1} \cdot \mathrm{cm}^{-1}\right)$. Upon irradiation with $313 \mathrm{~nm}$ light, the colorless solution of 1 a turned blue, in which the absorption maximum was observed at $557 \mathrm{~nm}\left(\varepsilon=8.9 \times 10^{3} \mathrm{~L} \cdot \mathrm{mol}^{-1} \cdot \mathrm{cm}^{-1}\right)$. Correspondingly, the blue colored solution returned to colorless upon irradiation with visible light $(\lambda>500 \mathrm{~nm})$, indicating that $\mathbf{1 b}$ returned to the initial open-ring isomer 1a. Similarly, upon irradiation $313 \mathrm{~nm}$ light, the color of dithienylethene 1b/PMMA film $(\lambda \max =275 \mathrm{~nm})$ changed from colorless to blue with the appearance of a new broad absorption band at $\lambda \max =575 \mathrm{~nm}$, which was assigned to the formation of the closed form $\mathbf{1 b}$. The red shift of the ring-closed form of diarylethene 1 in PMMA film in comparison with that in hexane solution can be ascribed to the stabilization of molecular arrangement in solid state.

\subsection{Fluorescence of Diarylethene 1a}

The fluorescence properties in hexane and in PMMA amorphous film of the compound 1a were measured using a Hitachi F-4500 spectrophotometer, and the breadths of excitation and emission slit were selected $10.0 \mathrm{~nm}$ and $20.0 \mathrm{~nm}$, respectively. As shown in Figure 2, the fluorescence intensity of diarylethene $\mathbf{1 a}$ decreased dramatically along with the photochromism from open-ring isomer to closed-ring isomer upon irradiation with $313 \mathrm{~nm}$ 


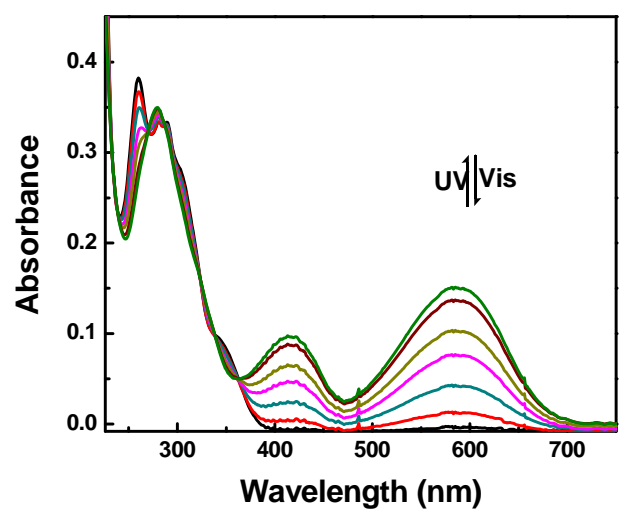

(a)

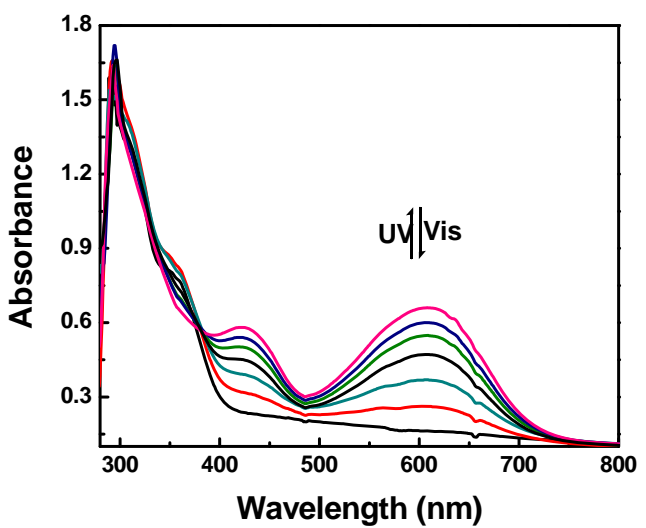

(b)

Figure 1. Absorption spectral changes of diarylethene 1 both in hexane and in PMMA film at room temperature: (a) in hexane $\left(\mathrm{c}=2.0 \times 10^{-5} \mathrm{~mol} \cdot \mathrm{L}^{-1}\right)$; (b) in PMMA film $(10 \%$, $\mathbf{w} / \mathbf{w})$.

light in hexane and in PMMA film. When irradiated by light of $313 \mathrm{~nm}$, the photocyclization reaction was carried out and the non-fluorescent closed-ring form of the compound was produced. The hexane solution and the PMMA film of 1a exhibited relatively strong fluorescence at 421 and $453 \mathrm{~nm}$ when excited at $355 \mathrm{~nm}$. The back irradiation by appropriate wavelength visible light regenerated its open-ring isomer and recovered the original emission intensity.

\subsection{Optical Storage}

The evaluation of potential of photochromic material as an erasable storage medium by recording, reading and erasing the optical image in a real-time operation was investigated. For the sake of long-term stability and environmental durability of storage devices, photoresponsive materials must be processed into large-area, high quality solid films. Usually, photochromic materials are doped into a polymer matrix for investigation of applications, and PMMA was chosen in this paper to be used as polymer matrix for optical recording. Using dithieny-

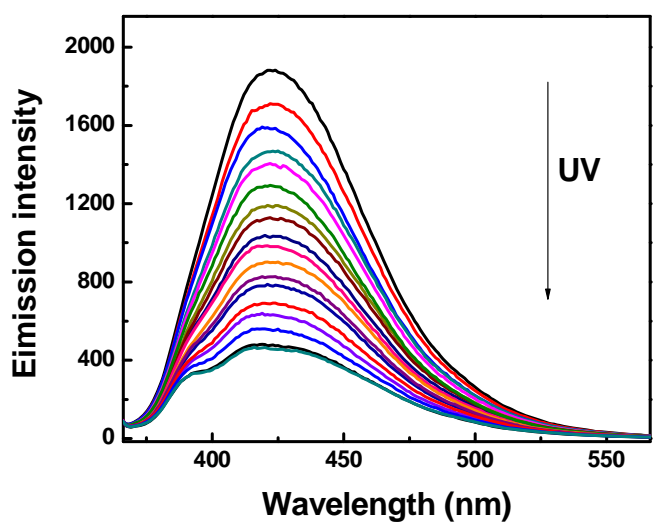

(a)

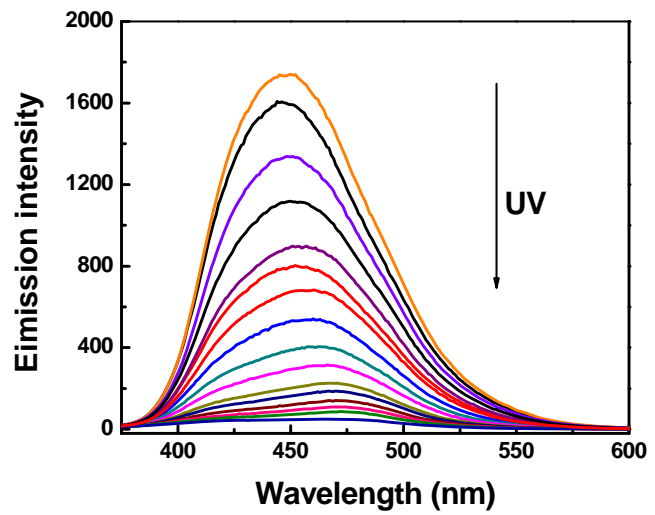

(b)

Figure 2. Fluorescent intensity changes of diarylethene 1(a) in hexane and (b) in PMMA film.

lethene 1c/PMMA as the recording medium, optical storage was performed by a $650 \mathrm{~nm}$ He-Ne laser. The film was colored homo-geneously by irradiation with UV light before optical recording. With a He-Ne laser in the experimental setup, the exposure time for each spot was $0.08 \mathrm{~s}$ and the patterns were about $20 \mu \mathrm{m}$. The polarizations of the patterns recorded on dithienylethene $1 \mathrm{~b} /$ PMMA film is shown in Figure 3. The result indicated that the new photochromic diarylethene can be applied in high capacity optical storage. As shown in Figure 3, the quality of optical recorded in 1c/PMMA film is also fairly good.

\section{Conclusion}

A novel photochromic diarylethene 1a was synthesized and its photochromic, reaction kinetics and fluorescent properties were investigated. The results showed that this compound exhibited good reversible photochromism both in solution and in PMMA amorphous film. It also exhibited remarkable fluorescence switching. Using diarylethene 1b/PMMA as recording medium, the results demonstrated that the diarylethene compound had attracttive properties for high capacity optical storage. 

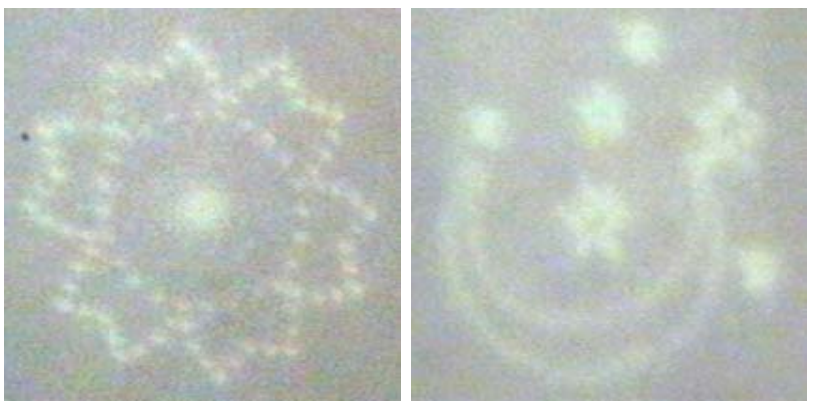

Figure 3. Readout patterns from the diarylethene 1c/ PMMA film.

\section{Acknowledgements}

A project supported by scientific research fund of Sichuan Provincial Education Department (10ZC063, 10ZC064, 12ZA146, 12ZB118).

\section{REFERENCES}

[1] Y. Chen, C. M. Wang, M. G. Fan, B. L. Yao and N. Menke, "Photochromic Fulgide for Holographic Recording," Optical Materials, Vol. 26, No. 1, 2004, pp. 75-77. doi:10.1016/j.optmat.2004.01.004

[2] S. Z. Pu, T. S. Yang, B. L. Yao, Y. L. Wang, M. Lei and J. $\mathrm{K}$. $\mathrm{Xu}$, "Photochromic Diarylethene for Polarization Holographic Optical Recording," Materials Letters, Vol. 61, No. 3, 2007, pp. 855-859. doi:10.1016/j.matlet.2006.06.084

[3] B. L. Yao, Y. L. Wang, M. Lei, N. Menke and G. F. Chen, "Polarization Patterns Hide and Display Using Photoinduced Anisotropy of Photochromic Fulgide," Optics Express, Vol. 13, No. 1, 2005, pp. 20-25. doi:10.1364/OPEX.13.000020

[4] N. Tanifuji, M. Irie and K. Matsuda, "New Photoswiching Unit for Magnetic Interaction: Diarylethene with 2,5-Bis(arylethynyl)-3-thienyl Group," Journal of the American Chemical Society, Vol. 127, No. 38, 2005, pp. 13344-13353. doi:10.1021/ja053200p
[5] M. Irie, "Diarylethene for Memories and Switches," Chemical Reviews, Vol. 31, No. 29, 2000, pp. 1685-1716. doi:10.1021/cr980069d

[6] H. Tian and S.-J. Yang, "Recent Progresses on Diarylethene Based Photochromic Switches," Chemical Society Reviews, Vol. 33, No. 1, 2004, pp. 85-97. doi:10.1039/b302356g

[7] T. Tsujioka, Y. Sesumi, R. Takagi, K. Masui, S. Yokojima, K. Uchida and S. Nakamura, "Selective Metal Deposition on Photoswichable Molecular Surfaces," Journal of the American Chemical Society, Vol. 130, No. 1, 2008, pp. 10740-10747. doi:10.1021/ja802430q

[8] D. Dulic, T. Kudernac, A. Puzys, B. L. Feringa and B. J. Wees, "Temperature Gating of the Ring-Opening Process in Diarylethene Molecular Switches," Advanced Materials, Vol. 19, No. 19, 2007, pp. 2898-2902. doi:10.1002/adma.200700161

[9] H. Tian and Y. L. Feng, "Nest Step of Photochromic Swiches," Journal of Materials Chemistry, 2008, pp. 1617-1622. doi:10.1039/b713216f

[10] S. Z. Pu, F. S. Zhang, J. K. Xu, L. Shen, Q. Xiao and B. Chen, "Photochromic Diarylethene for Three-Wavelength Optical Memory," Materials Letters, Vol. 60, No. 4, 2006, pp. 485-489. doi:10.1016/j.matlet.2005.09.021

[11] C. B. Fan, S. Z. Pu, G. Liu and T. S. Yang, "Substituent Position Effect on the Properties of Isomeric Photochromic Diarylethenes Bearing Chlorine Atoms," Journal of Photochemistry and Photobiology A: Chemistry, Vol. 194, No. 2, 2008, pp. 333-343. doi:10.1016/j.jphotochem.2007.08.032

[12] S. Z. Pu, H. H. Tang, B. Chen, J. K. Xu and W. H. Huang, "Photochromic Diarylethene for Two-Photon 3D Optical Storage," Materials Letters, Vol. 60, No. 29, 2006, pp. 3553-3557. doi:10.1016/j.matlet.2006.03.050

[13] B. L.Yao, Y. L. Wang, N. Menke, M. Lei, L. Y. Ren and S. Z. Pu, "Polarization Holograms Recording in Photochromic Diarylethenes Polymeric Film," Proceedings of SPIE, SPIE Press, Vol. 6343, No. 1, 2006, pp. 63432Z-163432Z-6. 